\title{
Le Chaos du fond du ciel selon Anaxagore
}

\author{
Maurice Caveing*
}

Résumé: Ce texte est un examen des Fragments du livre Sur la Nature d'Anaxagore de Clazomènes. On a écarté de l'enquête, par principe, l'étude des Témoignages et la discussion des très nombreux commentaires, anciens et modernes, qui constituent - et surchargent la tradition. Le retour aux textes mêmes - retraduits par nous - se fait dans la perspective d'une recherche du meilleur ordre que l'on peut assigner, avec vraisemblance, à ces Fragments. L'ordre proposé paraît cohérent, faisant se succéder quatre thèmes : le mélange primordial, la nature des choses séparées du mélange, l'état de composition des choses naturelles, le tourbillon cosmogonique. Chaque fragment prend ainsi sa place logique répondant à l'analyse de son contenu et l'ensemble situe la pensée anaxagoréenne dans l'histoire des cosmologies grecques des origines à Platon, après l'intervention de Parménide.

Abstract: This article examines the Fragments of the book On nature of Anaxagore of Clazomen. We let aside of the inquiry, as a matter of principle, the study of the Testimonies and the discussion of the many commentaries, ancient and modern, that constitute - and overload, the tradition. We come back to the texts - and translate them by our own - with the perspective of looking for the best likely order to be attributed to these Fragments. The propose order seems to be consistent, showing up a sequence of four themes : the primeval mixture, the nature of things separated from the mixture, the compound note of natural things, the cosmogonic whirl. Each fragment thus gets its logical place according to its content's analysis and the whole situates Anagore's thought inside the hisory of greek cosmologies from the origins to Parmenide and then to Plato.

QUE DANS CERTAINES conjonctures théoriques une cosmologie ne puisse s'édifier sans se coordonner d'étroite façon à une théorie de la matière, voilà ce qu'atteste fort bien l'histoire de la doctrine de la Nature dans la pensée grecque, du moins si l'on choisit une période pertinente. Les premiers "physiologues" présocratiques, il est vrai, n'ont pu élaborer suffisamment ni leur conception de la matière ni leurs idées cosmologiques pour que le problème d'une coordination entre elles puisse même se poser. Mais tout autre se trouve être la conjoncture après l'intervention de Parménide. La consistance logique de son ontologie moniste oblige ses successeurs, sauf à s'évader dans la sophistique, à rendre compte à la fois des phénomènes naturels temporalisés et de l'organisation cosmique. C'est pourquoi le moment spéculatif qui se déploie entre l'injonction de l'Eléate et les systèmes du monde de 1'Académie et du Lycée est

\footnotetext{
* Directeur de recherche, Centre National de la Recherche Scientifique.
} 

Fragment 1: ["Ensemble étaient toutes choses, infinies aussi bien en multitude qu'en petitesse: car le "petit" lui aussi était infini. Et, toutes étant ensemble, aucune n'était discernable, du fait de la petitesse; car toutes étaient maintenues en leur état par l'air et l'éther, qui sont tous les deux ensemble infinis; car ils sont ce qu'il y a de plus grand dans l'ensemble total, aussi bien en multitude qu'en grandeur.']

La célèbre formule par laquelle s'ouvre le texte est la réplique d'Anaxagore au "Il est, maintenant, tout ensemble, un" de Parménide (Frg. 8, v. 5-6), mais face au monisme éléatique, 1 "un" d'Anaxagore est une multiplicité infinie. Multiplicité de quoi? Le texte reste pour le moment dans la plus grande généralité: "toutes choses" Il faut pourtant éliminer toute acception "chosiste" du mot. De même, des termes comme "éléments", "substances", ne conviennent pas, ainsi qu'il sera clair par la suite. Le mot grec cependant n'est pas indifférent: cbrêmata, employé au sens vague: les choses, se distingue de pragmata, qui désignerait "les choses" au sens de "les affaires" On ne peut entièrenent faire abstraction du sens propre: les biens, les richesses, les choses qui constituent les ressources auxquelles on peut avoir recours, ici: les ressources dont dispose la Nature pour faire un monde.

Leur multiplicité est infinie, mais leur petitesse aussi. Par cette double référence au dénombrement et au volume, Anaxagore atteste que sa notion de l'infini, par rapport à l'emploi archaïque du terme, est déjà différenciée. Cette infinité en petitesse des choses primordiales - dont il est hors de propos, parce qu'anachronique, de demander s'il s'agit d'un infini potentiel à la manière d'Aristote - entraîne leur indiscernabilité dans leur "être-ensemble" La multiplicité initiale est donc une manière de continuum indistinct quant à l'apparence, mais non homogène quant à ses ingrédients.

Deux d'entre eux toutefois, même s'ils sont initialement indiscernables, jouent un rôle particulier. L'emportant à eux deux sur chacun des autres tant comme multiplicité que par l'immensité de l'étendue qu'ils occupent, ils maintiennent tous les autres dans leur état d'extrême division et donc de confusion. Ce sont l'Air et l'Ether. Ces deux principes sont bien connus de la physique ionienne. "Air" désigne l'atmosphère humide, lourde, chargée d'eau, formant brouillards et nuages. "Ether" désigne l'atmosphère sèche, légère, lumineuse, chargée de feu. L'un fait le ciel gris et l'autre bleu. Anaxagore les réunit tous deux pour les faire dominer sur "toutes choses" à l'état initial du mélange primordial. Celui-ci se présente donc, apparemment, comme une masse infinie et 
impalpable dans laquelle dominent l'air et l'éther, et baignent, à l'état de division illimitée, et indiscernables, toutes les autres "choses"

Fragment 3: [“”. car, pour le "petit", il n'existe certes pas de minimum de petitesse, mais il y a sans cesse à nouveau quelque chose de "moindre"(il n'est pas possible en effet pour l'étant de ne plus être) - mais, pour le "grand", il y a aussi sans cesse à nouveau quelque chose de "plus grand" Et le [grand] est égal au petit en multitude, et chaque [segment] est par rapport à soi aussi bien grand que petit."]

Voilà dans ce Fragment quelques-unes des précisions souhaitables. L'infinité de petitesse est justifiée. L'absence d'un minimum découle de la relativieé de la petitesse. Mais la descente infinie n'aboutit pas au néant, car l'être ne saurait se résoudre en non-être. La raison ainsi donnée est parménidienne. Rien de ce qui est ne saurait cesser d'être. Il y a donc un au-delà de la perception. ${ }^{3}$ L'infinité de grandeur paraît demander moins d'explication, en sorte que le réel anaxagoréen se déploie entre les deux infinis.

C'est alors que survient l'étonnant théorème: "le grand est égal au petit en multitude." L'accusatif de point de vue plêthos, "en multitude", est essentiel. Certes le "grand" n'est nullement égal au "petit" du point de vue de la grandeur, de la taille: ce serait une absurdité. Mais du point de vue de la multiplicité, il en va autrement. Il faut concevoir la multiplicité des constituants et redonner au mot grec isos (égal) son sens attesté d" "équivalent" Comment donc deux multiplicités, prises l'une dans le "grand", l'autre dans le "petit", seraient-elles équivalentes? Ce ne peut être qu'en raison de leur infinité. Ainsi Anaxagore professe-t-il qu'un tout infini est équivalent, quant à la multitude de ses constituants, à l'une de ses parties propres. Une structure granulaire de la matière qui impliquerait un minimum dans la petitesse ne saurait autoriser une telle conclusion, puisque tout volume serait un multiple entier du volume d'un granule. La matière anaxagoréenne est bien un continuum. Une partie quelconque, si infime soitelle, contient une multiplicité infinie de constituants, en équivalence avec toute autre partie, si grande soit-elle. Tout segment découpé dans ce continu (nous avons traduit en appuyant le neutre ékaston sur un substantif ajouté), envisagé sous le rapport de la multiplicité qui est en lui, est substituable à tout autre plus

3 Selon le Fragment 21 recopié par Sextus Empiricus, "nous ne sommes pas capables de distinguer la vérité à cause de la faiblesse de nos sens" 

en généralisant et en fixant la pensée propre d'Anaxagore. Des "semences" (spermata) de toutes choses, différentes de nature, et pour chaque nature en infinie multitude, voilà de quoi est fait le mélange. Il y a semence de la terre et de l'air..., du sec et de l'humide..., mais aussi de bien d'autres choses encore. Que les Opposés traditionnels des Ioniens soient représentés en eux-mêmes, mais sous cette forme, dans le mélange, n'empêche pas que les semences des autres choses, toutes différentes les unes des autres, soient qualifiées selon leur nature. La même remarque vaut pour la terre ou l'air: il n'y a pas, parmi les semences, d'espèces privilégiées jouant le rôle d"éléments" pour tout le reste. Enfin il ne semble pas que la terre soit pensée comme réductible à un mixte de froid et de sec: il n'y a pas synthèse de qualités opposées ou corrélatives dans des "éléments" Il semble exclu que les Opposés seuls - comme on l'a soutenu - soient semences des choses.

Compte tenu de ce que nous savons déjà sur la texture du mélange comme continuum partout indéfiniment divisible, les "semences" qui ne sont constituées que de leurs différences qualitatives, des unes par rapport aux autres, ne peuvent, semble-t-il, être conçues que comme des micro-régions, aussi petites que l'on veut, de ce continuum, mais néammoins différenciées d'espèce, parce qu'affectées de gradients qualitatifs qui déterminent leur spécification.

Nous savons maintenant ce que sont toutes ces "choses" qui sont "ensemble" dans la totalité du mélange primordial en son état initial, ces cbrêmata qui sont les ressources de la Nature. Ce sont des "semences" de tout ce que la Nature, dans son état actuel, comporte comme "choses" (en un second sens) différenciées, dans son inépuisable richesse de déterminations qualitatives. Mais ces choses naturelles, dont nous pouvons alors constater directement l'existence, ont été séparées (apokrinesthai) du mélange, et c'est notre second point.

\section{La vraie natute des êtres séparés}

Fragment 17: ["Les Hellènes ne conçoivent pas correctement la génération et la disparition: aucune chose en effet ne s'engendre ni ne disparait, mais à partir des choses préexistantes, elle se présente comme un mélange, puis elle se dissocie. Ainsi donc, ils parleraient correctement en appelant la génération "mélange" et la disparition "dissociation".]

Ce texte comporte un double usage du mot chrêma, "chose", au singulier et au pluriel. Au singulier il s'agit clairement de choses naturelles, données 
dans l'expérience, que d'autres Fragments désignent comme "choses séparées" Au pluriel il s'agit des choses dont il est question au Fragment 1, c'est-à-dire des semences invisibles formant le mélange primordial, qui constituent les ressources préexistantes de la Nature.

Nous devons aussi distinguer deux sortes de "mélange": le mélange primordial qui nous est désormais connu, et le mélange constitutif de chaque chose naturelle, dont il nous reste à apprendre de quoi et comment il est composé. Un point toutefois apparaît déjà clairement: c'est qu'une chose donnée ne provient pas d'une seule semence. Quant à la disparition, ce n'est que la dissociation (diakrinesthai) des composants du mélange en quoi la chose consiste.

Ainsi les "semences" sont-elles indestructibles et nul être ne peut provenir du non-être ni y retourner, conformément à l'injonction parménidienne. Le processus "mélange/dissociation" peut s'inverser en "dissociation/mélange" et, l'un succédant à l'autre, rendre compte des transformations observables dans la Nature. Le changement n'est en un sens qu'apparence et ne fait pas obstacle à la pérennité des semences, mais il n'en est pas moins réel: c'est une apparence fondée, puisque les composants indestructibles sont caractérisés par des déterminations et des qualités nullement illusoires.

Fragment 5: ["Ces dissociations s'étant ainsi produites, il faut savoir que le total [des choses] n'est de ce fait ni inférieur ni supérieur (car, qu'il y ait plus que le total, c'est impraticable), mais le total est toujours égal.”]

Le verbe correspondant à "dissociation" est encore ici diakrinesthai. Il est probable qu'il s'agit des dissociations qui affectent le mélange primordial lors de la formation des choses séparées. Le "total" n'est pas exactement le "tout" parménidien: celui-là est un singulier; ici nous avons un pluriel correspondant à la totalité des "choses" primitivement données, les "semences" originaires. Il ne saurait être question que leur totalité infinie s'accroisse. Mais l'on pourrait se demander si le processus de dissociation s'accompagne de perte de matière. Le présent texte nous avertit qu'il n'en est rien. Il y a toujours autant d'être, et le tout anaxagoréen, pour être divisible, est tout aussi égal à lui-même que le tout parménidien. Le principe éléatique est sauf.

Le thème qui se présente ensuite est celui de la nature du mélange constitutif des "choses séparées", c'est-à-dire de sa composition. La doctrine des "composés" se trouve être disséminée entre de nombreux Fragments. 


\section{Les composés}

Fragment 6: [“Alors, du moment que les parts du grand et du petit sont égales en multitude, il peut bien se faire que, de cette façon, en toute chose il y ait toutes choses; il n'est pas possible non plus qu'elles existent isolément, mais toutes ont une part de n'importe laquelle. Du moment qu'il n'est pas possible qu'il y ait un minimum, elles ne peuvent étre isolées ni subsister en soi, mais juste comme elles étaient au commencement, ainsi [sont-elles] encore maintenant, toutes ensemble. Elles sont présentes nombreuses et égales en multitude dans toutes les choses séparées, qu'on prenne celles-ci plus grandes ou plus petites."]

Dans ce texte nous rencontrons pour la première fois le terme moira, que nous traduisons de la façon la plus simple par le mot "part" Il est plus archaîque que meros: "partie" pour désigner la fraction $1 / \mathrm{n}$, c'est-à-dire "la n. part" Ce type de fraction est suffisant pour identifier les parts successives et de plus en plus petites obtenues par division illimitée sur un continuum. Il y a autant de telles fractions inférieures à $1 / 3$ qu'inférieures à $1 / 30$, à savoir une infinité, et pourtant, sur un segment donné de continuum, le trentième est plus petit que le tiers. Ainsi les parts du segment $1 / 3$ et celles du segment $1 / 30$ sont égales en multitude, c'est-à-dire quant à la multiplicité infinie qu'elles constituent. Ce sens fractionnaire de moira nous semble devoir guider l'interprétation et il est tout-àfait en harmonie avec l'arithmétique qui se pratiquait au temps d'Anaxagore. Il s'agit donc bien de cette équivalence en multitude du grand et du petit que nous avons analysée dans le Fragment 3 et qui ne peut s'entendre que d'un continuum. Faire dire au Fragment 6 que l'on peut diviser des choses inégales en un même nombre de portions, c'est le banaliser de façon stérilisante.

De cette équivalence des infinis, Anaxagore tire aussitôt la conclusion qu'il est possible qu'en tout segment du continuum se trouve représentée toute la variété séminale qui caractérise le mélange primordial. Or les "choses séparées" (et le verbe employé dans ce cas est apokrinestbai) ne sont rien d'autre que de tels segments qui se sont détachés du mélange. D'où l'assertion qu'il y a chance qu'en toute chose (au singulier) il y ait toutes choses (au pluriel), c'est-àdire toutes les ressources de la Nature sous forme de "semences" Bien entendu, s'il en est ainsi, il reste à expliquer ce qui distingue entre elles les "choses séparées" dont la Nature nous offre le spectacle. Mais n'anticipons pas! 
$A$ contrario, le texte argumente la thèse que ces semences ne peuvent exister isolément (chôris) ou être isolées (chôristhènai). Si en effet l'une quelconque de ces semence subsistait isolément en soi, cela signifierait qu'elle ne peut être divisée en deux ou plusieurs autres, c'est-à-dire qu'aurait été atteint un minimum de petitesse. Or cela est exclu, comme nous l'avons vu aux Fragments 1 et 3. Par conséquent une semence ne peut exister qu'en association avec d'autres dans le continuum qu'elles forment par leur "être-ensemble", ou alors immergée dans un de ses segments quel qu'il soit. Nulle part d'ailleurs dans les textes dont nous disposons le mot n'intervient autrement qu'au pluriel (spermata).

La seconde phrase du texte: “il n'est pas possible non plus qu'elles existent isolément, mais toutes ont une part de n'importe laquelle" est suspecte. D'une part elle forme doublon avec la phrase suivante qui est tout-à-fait claire et que nous venons d'expliquer; d'autre part elle est grammaticalement inconsistante, car l'impossibilité de l'existence isolée doit être dite de toutes ces choses que sont les chrêmata du mélange originaire, c'est-à-dire les semences, tandis que les choses dont on dit que "toutes ont une part de n'importe laquelle" sont les "choses séparées" qu'on trouve dans la Nature. Pareille inadvertance trahit la glose marginale qui se serait glissée dans le texte.

La dernière phrase confirme que les choses séparées, quelle que soit leur taille, contiennent toutes et chacune la multitude infinie des choses présentes "toutes ensemble" dans le mélange primordial, c'est-à-dire une représentation de toutes les sortes de semences.

Fragment 7: [“... de sorte qu'on ne peut connaitre la multitude des choses séparées ni par raisonnement, ni par expérience."]

A propos des choses séparées (apokrinesthai), ce Fragment note que leur multiplicité dans la Nature n'est déterminable ni par raisonnement ni par expérience. Il semble qu'il s'agisse des individus, non de leurs genres, distinction qui d'ailleurs n'apparaît pas dans les Fragments dont nous disposons. Mais l'infinité de la réserve séminale naturelle, le mélange originaire indestructible, garantit la reproduction de la Nature par une succession numériquement inassignable d'êtres séparés.

Fragment 4, Iè. partie: ['S'il en est bien ainsi, il faut penser que dans tous les composés il y a multiplicité et diversité, c'està-dire des semences de toutes choses, ayant des aspects, des couleurs et des qualités sensibles variés...[ suit un passage concer- 
nant la formation des hommes et des vivants en général, du Soleil, de la Lune, et des autres astres, la production des fruits de la Terre.] Voilà ce que j'ai professé au sujet du processus de séparation, à savoir qu'il n'y aurait pas eu séparation seulement chez nous, mais aussi ailleurs." [suit la 2è. partie du Fragment 4, q.v-supra.]

Les "composés" sont désignés par le participe passé de synkrinestbai; "séparation" est apokrisis, correspondant à apokrinesthai, déjà rencontré dans la 2è. partie du Fragment, ainsi qu'aux Fragments 6 et 7. Nous avons donc ici le texte qui établit la corrélation entre la séparation et la composition: les choses séparées du mélange initial sont des choses composées, c'est-à-dire formées à partir des semences qui sont dans le mélange. Le second point à souligner, c'est que ce texte met particulièrement en relief le caractère qualitatif des déterminations séminales: les semences sont extrêmement variées dans leur aspect, leur couleur et leurs autres qualités sensibles; "qualité" est exprimé par le terme bédonè qui évoque les plaisirs des sens. Rien de ce qui fait la richesse de la Nature n'est donc perdu dans la conception d'Anaxagore. Il faut bien garder à l'esprit toutefois qu'avant la séparation, ces qualités sont imperceptibles et les semences indiscernables en raison de leur petitesse et de leur mélange.

D'autre part l'emploi de deux verbes distincts: apokrinestbai et diakrinesthai, apparu aux Fragments 17 et 5, ne permet pas de confondre la séparation et la dissociation. Le Fragment 17 avertit que la dissociation est le processus inverse d'un mélange, c'est-à-dire de ce que le présent texte permet de nommer: "composition" On peut donc supposer que le processus de séparation, par lequel un fragment du mélange initial s'en distingue, est précédé d'un processus par lequel certaines semences de ce mélange en sont dissociées, et accompagné du processus de leur (re)composition dans la chose naturelle en voie de séparation.

La dernière phrase du présent texte ouvre un débat parmi les érudits sur la question de savoir si Anaxagore professait la pluralité des mondes à partir du mélange primordial.

Fragment 12, dernière phrase: [“Aucune autre chose n'est jamais semblable à aucune [autre], mais ce dont il y a le plus en elle, c'est cela - le plus discernable en elle - qu'elle est et a été, chacune en son individualité.'] 
Nous isolons cette phrase afin de compléter dès maintenant la conception des choses composées et séparées, sans cela inintelligible. Il s'agit ici en effet de toutes les choses de la Nature, à l'exception de l'Esprit, ou Intelligence, dont nous verrons l'intervention ci-après.

Le texte lève la difficulté de concevoir comment se distinguent les unes des autres des choses toutes formées pourtant d'une infinie multitude de semences. La solution est dans la proportion majoritaire de certaines semences sur toutes les autres, présentes, mais très faiblement représentées. Il y a dans chaque chose de la Nature certaines qualités (ou faut-il dire "sortes"?) de semences dominantes et d'autres récessives. Ce qu'une chose est par essence (car tel est le sens de la redondance gnomique: "est et a été") c'est simplement "ce dont il y a le plus en elle"

Or c'est aussi ce qui, en elle, est "le plus discernable" L'indiscernabilité des semences dans le mélange originaire était l'effet de leur dispersion et de leur petitesse. Dans une "chose séparée" de la Nature, des semences semblables, voire identiques, sont regroupées massivement: elles sont alors dominantes et leurs propriétés qualitatives deviennent perceptibles. De même, des points colorés dispersés demeurent invisibles, tandis que, regroupés de façon adéquate, ils forment des plages colorées qui composent une image parfaitement visible, et pourtant formée uniquement de points. Les caractères dominants confèrent à la chose son individualité, en sorte qu'aucune n'est jamais semblable à aucune autre; de légères variations dans la dominance permettent donc de prendre en compte les particularités individuelles. Quant aux caractères "récessifs" en quelque sorte, ils correspondent à des semences si dispersées dans la masse que leurs qualités, et donc leur présence, demeurent inaperçues. Néanmoins chaque chose contient, à sa façon propre, l'infinie multiplicité des semences universelles.

On voit donc en quoi consiste le processus dissociation/composition qui s'opère au sein du mélange initial juste avant la séparation d'un être naturel. Il s'agit d'un réarrangement des semences qui vont le composer: elles se dissocient de leurs voisines dans le mélange et se regroupent pour former un mélange spécifié et séparable, désormais à dominante.

Fragment 11: ["En toute chose il y a une part de toute chose, excepté de l'Esprit, mais il y en a dans lesquelles l'Esprit aussi est présent.']

Mis à part le cas spécial de la présence de l'intelligence dans certains êtres naturels, le Fragment affirme une loi universelle de la Nature: "en toute chose il y a une part de toute chose", dont nous pouvons saisir maintenant en 
quel sens il faut la comprendre. En effet, il y a en toute chose une multiplicité infinie de semences qui n'y sont pas dominantes, mais qui sont dominantes les unes dans telle autre chose, les autres dans telle autre, et ainsi de suite pour toutes les autres choses de la Nature, en sorte que, dans la chose considérée, il y a une part (moira) de chacune des autres choses, puisque le continuum se divise en fragments aussi petits que l'on veut, sans qu'il existe jamais de semence isolée. Ainsi toute chose contient une part infime et indiscernable de ce qui fait l'essence de chacune des autres choses, mais qui ne constitue pas son essence à elle. Une autre interprétation du texte, qui ferait de toute chose, par essence, un magma de tout ce qui fait l'essence des autres choses, serait absurde.

D'une chose à une autre - de nature différente, faut-il ajouter - il n'y a donc aucune similitude dans la répartition interne des semences constitutives: chaque chose dans la Nature, en quelque sorte, a sa formule, mais il est bien entendu que les semences qui y sont dominantes le sont partout, c'est-à-dire en toutes ses parties, en vertu du principe de la divisibilité à l'infini et de l'équivalence du "grand" et du "petit" De manière analogue, l'or impur n'en est pas moins de l'or en toutes ses parties.

Fragment 10: ["Car comment du poil proviendrait-il de ce qui n'est pas poil, et de la chair de ce qui n'est pas chair?”]

Il n'est pas absolument certain qu'on ait ici les propres termes d'Anaxagore, car ce Fragment est tiré d'un scolie à Grégoire de Nazianze: sa transmission est donc anonyme; cependant il est présenté comme résumant en une formule imagée le commentaire assez abondant qui l'entoure.

Le texte exprime le principe parménidien que 1'être ne peut provenir du non-être, mais il le fait de façon concrète, sur le cas de choses spécifiées qualitativement. Si le poil ou la chair se forment et apparaissent chez les animaux, ce ne peut être qu'à partir de choses dans lesquelles les semences du poil ou de la chair étaient bien présentes, encore que peut-être récessives et inapparentes. Il y a ainsi en toute chose de quoi en former d'autres par dissociation et recomposition, lesquelles seront des "mélanges à dominante" différente, d'essence différente. Il semble donc qu'au cours des cycles naturels des semences puissent transiter, à l'état récessif, à travers des choses très diverses, avant de parvenir à devenir dominantes dans la chose dont elles déterminent l'essence. ${ }^{4}$

4 Le terme "homéomère" répandu par Aristote à propos de la doctrine de la Nature d'Anaxagore ne se trouve nulle part dans les Fragments; il ne figure pas non plus dans les textes de Platon ni de 
Fragment 21a: [“Ce qui apparait est en effet un aperçu sur l'invisible."]

Ce texte fait état explicitement des "phénomènes" (phainomena), au sens de "ce qui se montre", "ce qui apparait", et indique le rapport entre ce qui apparaît et ce qui est. Ce qui est en dernière instance, c'est-à-dire le domaine des semences, demeure invisible du fait de leur petitesse et de la faiblesse de nos sens. Nous ne percevons à l'état d'association, et non en elles-mêmes, que celles qui sont dominantes dans une chose donnée, et nous ignorons l'existence des autres. En un sens, le phénomène n'est donc pas illusion; il est bien un aperçu de ce qu'est la chose en elle-même, une manifestation de son essence. Mais, s'il nous permet d'identifier la chose, ce n'est pas lui qui nous apprend la raison pour laquelle c'est bien son essence qui se manifeste ainsi, à savoir que celle-ci consiste dans la dominance de certaines semences et que leur association de ce fait est devenue visible. Ainsi la coupure parménidienne entre l'être et l'apparence est résorbée: la distinction du phénomène et de l'essence est maintenue, car l'essence n'est connue que par raisonnement, mais le phénomène est fondé dans l'essence: étant donné ce qu'est la chose, elle ne peut apparaître autrement qu'elle apparait. La richesse et la variété des apparences est fondée dans la nature des choses.

Ce Fragment clôt la série qui est consacrée aux "composés" séparés du mélange initial. Toutefois, si ont été identifiés et caractérisés les processus qui doivent permettre de passer de celui-ci à ceux-là, en revanche nous ne savons encore rien de la nature et de l'origine du mouvement par lequel s'effectuent ces processus. C'est l'objet du quatrième thème.

Xénophon se rapportant à Anaxagore. "Homéomère"se dit d'un tout qui est semblable à ses parties, ainsi en Géométrie la ligne droite, la circonférence de cercle, l'hélice cylindrique; et dans la biologie d'Aristote, les divers tissus animaux ou végétaux: chair, os, sang, nerf, bois. Comme à son habitude Aristote désigne des choses mentionnées par Anaxagore au moyen de son propre vocabulaire. Ces "choses" peuvent être considérées comme "homéomères' pour deux raisons, semble-t-il, la première étant l'équivalence du "grand" et du "petit" en tant qu'infinies multiplicités, donc du tout et de la partie, la seconde étant qu'Anaxagore paraît individualiser les "choses séparées" surtout qualitativement, en sorte que les organismes complexes, par exemple les animaux, sont formés de ces choses par assemblage (sympegnusthai: Fragments 4 et 16)et que, dans ce cas, elles s'identifient aux "homéomères" d'Aristote. 


\section{La révolution cosmogonique}

La réponse à la question du mouvement se trouve dans le Fragment 12, célèbre en raison du rôle qu'il donne à l'Esprit, sur lequel principalement a insisté la tradition métaphysique. Mais ce rôle est inséparable de la mise en ordre cosmique, en sorte qu'il faut citer le Fragment tout entier.

Fragment 12: ["Les autres[choses] ont en elles une part de toute chose, tandis que l'Esprit est sans limites et son propre maître: il ne se mélange à aucune chose, mais demeure seul luimême en lui-même. Car s'il n'était pas en soi, mais se mélangeait avec quelque chose d'autre, il participerait de toutes les choses en se mélangeant à l'une d'elles: car en toute chose se trouve une part de toute chose, comme je l'ai dit précédemment. Mais les choses auxquelles il serait mélangé l'empêcheraient d'être le maître d'aucune d'entre elles, comme il l'est en étant seul en soi. Car il est entre toutes les choses la plus subtile et la plus pure, et il détient véritablement sur toute chose la connaissance complète et la puissance la plus grande; et tout ce qui possède vie, qu'ils soient plus grands ou plus petits, tous l'Esprit en a la maîtrise. Et la rotation dans sa totalité, l'Esprit en a eu la maîtrise, de telle sorte qu'il a donné l'impulsion au [mouvement de] rotation. Et d'abord il fit débuter la rotation à partir d'une petite [région], puis la rotation s'étend, et s'étendra encore davantage. Et les choses à l'état de mélange et celles qui se séparent et celles qui sont dissociées, toutes l'Esprit en a eu la connaissance. Et les choses qui devaient venir à l'être, quelles qu'elles fussent, et qui furent, mais ne sont plus maintenant, et celles qui sont maintenant, quelles qu'elles soient, et celles qui seront, toutes l'Esprit les a soumises à l'ordre cosmique, y compris cette rotation dans laquelle tournent actuellement les astres, le Soleil, la Lune, l'air et l'éther, qui sont séparés.

C'est la rotation elle-même qui produisit la séparation. Alors du rare se sépare le dense, du froid le chaud, du sombre le lumineux, de l'humide le sec. Et d'une multitude de choses il y a une multitude de parts. Mais de façon absolue, rien ne se sépare ni n'est dissocié l'un de l'autre, excepté 1'Esprit. L'Esprit est tout entier semblable [à soi-même], qu'il soit plus grand ou plus petit... [suit la dernière phrase, q.v.supra]'] 
Ce Fragment contient la plus ample énumération des attributs de l'Esprit, ce principe qu'Anaxagore est le premier à introduire dans l'histoire des cosmologies grecques, du moins sous cette forme explicite. Une première série d'attributs le différencie de façon décisive de tous ceux qu'avaient pu invoquer les penseurs antérieurs, notamment ioniens. Ce sont ceux qui creusent l'écart entre lui et toutes les autres "choses" de la Nature. Il est pur de tout mélange, ni mélange en lui-même, ni se mélangeant à quoi que ce soit d'autre; il est donc absolument séparé et, partant, il est libre au sens de sans entraves. A la différence des "choses séparées" du mélange primordial, il est sans limites et, sans que ce soit dit, unique. Par conséquent il est en soi et cause de soi. Son éternité est affirmée dans le Fragment 14 (ci-après). Deux attributs fondamentaux le caractérisent en lui-même: il est tout-connaissant et tout-puissant. A ce titre il est premier moteur et principe de l'ordre cosmique. Enfin il est coextensif au mélange primordial et au Cosmos qui s'en distingue progressivement, et dans toute cette étendue il demeure partout semblable à soi, simple et homogène.

Ainsi bien qu'il y ait chez Anaxagore séparation totale entre d'une part le mélange primordial dans son infinie multiplicité indistincte, et de l'autre l'Esprit illimité, unique et simple, il est difficile de dire qu'il y a opposition entre le "matériel" et "l'immatériel" Ces catégories ici ne sont pas encore opératoires. L'Esprit qui est "la plus subtile" des choses (cbrêmata) est en quelque sorte joint à toutes les parties de l'étendue, et c'est sans doute ce qui lui permet de les "connaître" et en même temps de leur communiquer le mouvement qui ne vient que de lui seul. En outre il n'a aucun attribut moral: la Nature d'Anaxagore n'est ni bonne ni mauvaise. Nulle part à son sujet le mot “dieu” n'est prononcé, sans doute pour le distinguer absolument des personnages mythologiques. Enfin, en ce qui concerne les êtres de la Nature chez qui, selon le Fragment 11, l'Esprit aussi est présent, il est probable qu'il y est joint en même façon, en sorte qu'à la fois il leur procure la connaissance et les anime. Le fait qu'il soit semblable, aussi bien petit que grand, garantit, semble-t-il, la possibilité d'une connaissance rationnelle du Cosmos par l'homme, puisque partout c'est la même Intelligence qui connait.

Le texte confirme les états ou processus dans lesquelles les ressources initiales de la Nature (chrêmata) peuvent se trouver ou être engagées: à l'état de mélange, en voie de dissociation (diakrinestbai) ou de séparation (apokrinestbai). Tous sont sous le contrôle de la connaissance qu'en a l'Intelligence cosmique. Il en résulte le devenir dans le temps des "choses séparées", dans leur diversité qualitative et leur multiplicité quantitative, toutes soumises cependant dans la simultanéité et dans la durée à l'ordre déterminé par cette Intelligence. 
Sur ces questions, la nouveauté du Fragment est d'introduire le tourbillon cosmogonique, c'est-à-dire comme mode de formation du Cosmos. Cette rotation a reçu le branle de l'impulsion initiale que lui a donnée l'Esprit. Même si l'on sait qu'alors "toutes choses étaient ensemble", on ne sait rien sur l'éventuelle durée antérieure de ce mélange indistinct. Avec l'impulsion de l'Esprit, c'est l'Univers lui-même qui commence d'exister et avec lui s'instaure un temps universel à partir d'un instant initial (ce que marque dans le texte l'apparition du passé, du présent et du futur du verbe "être"). Cependant la durée écoulée depuis cet instant reste indéterminée et inconnue.

La rotation dans le mélange primordial débute en un point, puis elle accroît son rayon et élargit de proche en proche son domaine, en sorte que le Cosmos s'accroît et s'accroitra encore indéfiniment aux dépens du mélange primordial, dans une sorte de bulle sphérique en expansion à l'intérieur de l'infinité du mélange qui se trouve toujours à sa périphérie. Géométriquement cela n'est possible que par la rotation autour d'un diamètre (axe des pôles) d'un cercle (méridien) dont le rayon croit de zéro à l'infini, mais Anaxagore n'en dit rien. ${ }^{5}$ La question de savoir si le mouvement se poursuit de lui-même ou est toujours entretenu par l'Esprit n'est ni précisée ni tranchée. C'est cette même rotation qui emporte actuellement dans le mouvement diurne les astres, le Soleil, la Lune, l'air et l'éther, qui sont ici donnés comme séparés (apakrinestbai) alors que, dans le Fragment 1, ils étaient encore dans le mélange primordial comme ce qui y était le plus répandu et dominant.

C'est la rotation qui est responsable de la séparation, sans doute parce que le tourbillon arrache de proche en proche des semences d'une certaine sorte qui se regroupent en lui pour former une "chose séparée" un "mélange à dominante" Il semble que la séparation affecte d'abord les Opposés ioniens, qui reparaissent ici: le dense, le froid', le sombre et l'humide, qui caractérisent l'air, par opposition au rare, au chaud, au lumineux, au sec, qui caractérisent l'éther. Il se forme ainsi une multitude de parts (moira) d'une multitude de choses, c'està-dire des groupemements formés de semences agglutinées entre elles et dissociés les uns des autres, promis à d'ultérieures recompositions. Cependant, prise parmi les choses issues du mélange, aucune séparation ni dissociation ne doit être entendue de façon absolue, c'est-à-dire sans doute ni complète ni défini-

5 Deux témoignages laissent entendre cependant qu'il connaissait l'inclinaison de l'axe des pôles, qu'il attribuait, croyant la Terre plate, à un basculement ultérieur de 1'Univers: Diogène Laërce, II,9 = $D K^{6}, 59$ A 1, p. 5-6, et Aétius, 11,8,1 = DK, 59 A 67, p. 22 (DK ${ }^{6}$ II abrège: DIELS \& KRANZ: Die Fragmente der Vorsokratiker (6è. éd., vol. II). 
tive. Le continuum qui se différencie en lui-même et dont certaines choses deviennent bien distinctes, n'est pourtant jamais rompu. Seul l'Esprit est absolument à l'écart de tous ces processus. Les Fragments suivants vont d'ailleurs fournir d'autres précisions.

Fragment 13: ["Et quand l'Esprit commença le mouvement, il était séparé de tout ce qui était en mouvement, et ce que l'Esprit mit en mouvement, tout cela, en même quantité, fut soumis à dissociation; et la rotation des choses en mouvement et en voie de dissociation accrut beaucoup plus encore le processus de dissociation."]

Deux précisions en effet ressortent de ce texte: d'une part la rotation n'est pas responsable seulement de la séparation, mais aussi bien de la dissociation (diakrinesthai): par la turbulence qu'il introduit dans le mélange originaire, le mouvement dissocie les semences les unes des autres, sans que cela se solde par une perte de matière; d'autre part il semble que cet effet dissociatif de la rotation s'amplifie encore de façon cumulative, d'une manière purement mécanique, en ce sens que les choses en mouvement et en train de se dissocier en entrainent sans cesse davantage d'autres dans leur tourbillon: l'Esprit n'aurait donc même plus à intervenir.

Fragment 9: [... [ces choses] étant ainsi soumises à la rotation et à la séparation, par l'effet de la force et de la vitesse. C'est la vitesse qui produit la force. Mais leur vitesse ne ressemble en rien à la vitesse des choses actuellement existantes parmi les hommes: au total, elle est de multiples fois plus rapide."]

Nous avons la confirmation de l'effet mécanique du mouvement, qui produit la séparation (apokrinesthai) par l'intermédiaire de la force. De plus les vitesses cosmiques ne sont pas de l'ordre de ce qui peut être constaté sur Terre; il faut les concevoir comme des multiples qui font sortir du registre auquel l'homme est habitué.

Fraqment 8: ["Les [choses] qui sont dans le Cosmos [qui est] un ne sont pas isolées les unes des autres, ni coupées à la hache, ni le chaud du froid, ni le froid du chaud."] 
Ce texte rappelle, dans l'intérêt de l'unité du Cosmos, la non-séparation absolue des choses de la Nature, déjà affirmée au Fragment 12. D'une part il n'y a pas de vide entre les choses; d'autre part les qualités s'interpénètrent, l'exemple donné étant celui de la présence relative des Opposés l'un dans l'autre, le chaud dans le froid, le froid dans le chaud, assurant la gradation insensible de l'échelle des températures, ou plus généralement des "grandeurs intensives."

Les Fragments suivants vont permettre d'identifier quelques étapes de la formation du Cosmos.

Fragment 2: [“... Car air et éther se séparent de la multitude qui les enveloppe, et cette enveloppe est certes une multiplicité infinie."]

L'air et l'éther dominants dans le mélange originaire selon le Fragment 1 et donnés comme séparés au Fragment 12, apparaissent ici comme les premiers à se séparer (apokrinestbai) du mélange, formant à l'intérieur de celui-ci la bulle cosmique promise à s'étendre indéfiniment. Le mélange subsiste alors à la périphérie de la bulle et devient son enveloppe (périéchon). Il est d'ailleurs réaffirmé aussitôt que, comme il a été dit au Fragment 1, ce mélange désormais enveloppant est infini quant à la multitude dont il est composé. Comme il est infini, l'expansion de la bulle cosmique, qui s'effectue, ne l'oublions pas, à ses dépens, ne l'affecte en rien quant à son étendue, à son inépuisabilité, ni à sa capacité enveloppante. On peut donc considérer que cette "enveloppe" forme depuis l'origine des temps le "fond du Ciel" d'Anaxagore.

Fragment 15: ["Le dense, l'humide, le froid et le sombre se rassemblèrent là-même où maintenant [se trouve la Terre], tandis que le rare, le chaud, le sec, [le lumineux] émigrèrent aux confins de l'éther."]

Cette seconde étape est constituée par la dissociation (relative) du complexe air/éther et sa recomposition en éther d'une part, air de l'autre, par la séparation (relative) des Opposés dans chacun des couples, déjà évoquée au Fragment 12 , suivie de leur regroupement: la première série qualitative se rassemble là où ils se trouvent maintenant, au voisinage de la Terre, tandis que la seconde émigre aux confins du Cosmos, là où commence l'éther. A cette étape, le Cosmos est donc, à partir de l'enveloppe, étagé en deux couches: l'une d'éther entourant un noyau central d'air, le tout bien sûr sous 1'effet de la rotation cosmi- 
que. Il apparaît ainsi que dans le tourbillon les parties denses ont un mouvement centripète.

Fragment 16: ["[Formée] à partir de ces [choses] en cours de séparation la Terre se solidifie; en effet des nuages se sépare l'eau, de l'eau la terre, et à partir de la terre les roches se solidifient sous l'effet du froid, mais ces dernières s'en échappent mieux que [ne le fait] l'eau.']

Dans cette troisième étape, le mouvement centripète de différenciation se continue. L'air, qui est dense, humide, froid et sombre, compose les nuages, dont se sépare (apokrinestbai) l'eau. Dans une quatrième étape, la terre se sépare de l'eau, toujours en se portant vers le centre. Les roches sont des parties solidifiées de la terre, elle-même formée par compactification de choses antérieurement séparées: des poussières terreuses seraient en suspension dans l'eau comme l'eau est en suspension dans l'air. Mais les roches ont une destinée surprenante, car elles s'échappent de la Terre mieux que l'eau, et s'il faut en croire le témoignage d'Hippolyte, le Soleil, la Lune et tous les astres sont des roches enflammées qui sont emportées circulairement par la révolution de l'éther, le Soleil étant sous les étoiles, la Lune sous le Soleil, et d'autres corps encore en-dessous, invisibles pour nous, entrainés dans la même révolution ${ }^{6}$ Il y a là, semble-t-il, un mouvement centrifuge par lequel les roches s'échappent de la Terre, à l'encontre du mouvement centripète qui produisait condensation et concentration. Peutêtre est-ce un exemple de la non-séparation radicale de deux processus opposés, à quoi d'ailleurs s'ajoute que des fragments rocheux peuvent se détacher de ces corps et retomber sur Terre, comme dans le cas du fameux aérolithe de l'Ægos Potamos, qu'Anaxagore aurait déclaré provenir du Soleil ${ }^{7}$

Fragment 14: ['L'Esprit, qui toujours existe, subsiste en vérité maintenant comme toujours là où se trouve aussi tout le

6 Hippol., Refut., I,8,1sq. $=D K^{6} \mathrm{II}, 59$ A 42: 6, p. 16, 1. 16-19, et 7, p. 16, 1. 21-22. En fait Hippolyte déclare ensuite que la Lune n'a pas de lumière propre, mais la reçoit du Soleil (ibid., 8, p. 16, 1. 18), qu'elle est faite de terre et comporte des plaines et des ravins (ibid., 10, p. 16, 1. 29) et qu'Anaxagore a expliqué les éclipses (ibid., 9-10): la Lune ne semble donc pas enflammée. Quant à la Voie Lactée, elle est due à la réflexion de la lumière des étoiles non éclairées par le Soleil parce que se trouvant dans l'ombre du disque terrestre (ibid., 10, p. 16, 1. 30-1).

7 Diogène Laërce II, $10=D K^{6}$ II, 59 A 1, p. 6, 1. 9-10, et Pline, N.H., II, $149=D K^{6}$ II, 59 A 11, p. 9 , 1. $16-20$. 
sens précis que l'on peut dire de type mathématique, ne peut pas ne pas avoir gardé, à l'époque d'Anaxagore, à titre de connotation, quelque chose de son sens ancien qui évoque l'absence de détermination, de limites et de repères, donc une région que l'on ne saurait franchir.

Par là se retrouve chez Anaxagore, sous une forme qui lui est propre, un prolongement d'une tradition de la pensée cosmogonique grecque, dans laquelle Chaos et apeiron ont partie liée. Alors que le Chaos hésiodique est ouverture béante (chainein), gouffre qui s'entr'ouvre, espace qui engloutit, une cosmogonie poétique du VIIè siècle (BC) évoque dans le même sens l'image de la haute mer par nuit de tempête et de brouillard, sans lune, où le marin, privé de tout repère terrestre et stellaire, ne peut qu'errer interminablement, prisonnier de l'insondable Nuit au milieu des éléments déchainés: l'océan apparaît ici comme l'étendue abyssale et chaotique, espace infranchissable où s'efface aussitôt toute trace du chemin suivi. Dans cette image, sont présentes bon nombre des entités mythologiques qui seront, au gré des diverses cosmogonies, des avatars du Chaos: Okeanos, l'Erèbe, l'Air brumeux, la Nuit, de laquelle dans certains textes procède I'Ether. Or ces entités sont en même temps des figures de ce qui est infranchissable parce que privé de chemins, de limites, de repères, d'orientation, bref des figures de l'apeiran. ${ }^{11}$

L'association des deux thèmes se poursuit chez les "physiologues" Aristote suggère que l'Eau primordiale de Thalès a pour ancêtre mythique Okeanos et que l'Air d'Anaximène doit être dit apeiron. ${ }^{12}$ C'est Anaximandre qui, le premier, choisit le terme abstrait lui-même (apeiron) pour désigner l'état-de-choses primordial, l'Indéterminé, puissance originaire antérieure à toute détermination, donc Chaos, antérieur à la différenciation des opposés, enveloppant entièrement la Terre et les Cieux, immense, éternelle et infranchissable nuit extérieure, abîme insondable où nul ne peut jamais s'aventurer.

Chez les Pythagoriciens, apeiron comme principe rencontre un principe opposé: peras, la limite, le repère. Le couple Indéterminé/Détermination évolue vers le couple Illimité/Limite. Le Cosmos naît de l'articulation des deux principes, mais baigne dans l'apeiron extérieur, indéterminé et illimité. Le Cosmos com-

11 Sur tous ces points, cf. M. CAVEING, La Figure et le Nombre. Lille, Presses Universitaires du Septentrion, 1997, Cap. VI, 3, 1, p. 278-86. La racine de apeiron, peras, a en effet archaïquement une double signification: "chemin" et "lien". Notons qu'appliqué à l'Esprit, le terme signifie que l'Esprit est au-delà de toute entrave, de toute limitation, qui pourrait réduire sa connaissance et sa puissance.

12 Aristote: Met., I, 983b6-984a2; Du Ciel, II, 13, 294a28-b1; Met., XI, 1066b21; Pbys., III, 4, 203a16; III, 5, 204b25. 
mence avec l'Un, autre nom de la Limite, qui s'implante dans l'apeiron et s'y développe en déterminant l'indéterminé.

Toutes ces cosmogonies, qu'elles soient mythiques ou spéculatives, sont autant de raisons de penser que, lorsqu'Anaxagore décrit le mélange primordial comme infini, il faut l'entendre aussi bien comme illimité dans le grand et le petit, comme infiniment multiple quant à ses composants, que comme indéterminé en ce que "toutes choses" y sont confondues et indiscernables, et que le continuum ne comporte aucune démarcation. Rien n'y est distingué: c'est donc bien une nouvelle figure du Chaos des origines, et ce n'est pas la présence en lui de l'air et de l'éther qui peut y faire obstacle, puisqu'ils sont eux-mêmes présents sous la même catégorie dans plusieurs cosmogonies. La particularité du Chaos anaxagoréen, c'est qu'il est explicitement riche: il est le réservoir inépuisable de toutes les ressources de la Nature, la source surabondante de l'infinie diversité du monde.

Le renversement qu'opérera Platon se manifeste clairement dans le contraste avec la manière dont l'indéterminé intervient dans le Timée. Espèce difficile et obscure à concevoir puisqu'il est la négation de toute détermination, il est ce dans quoi peut prendre naissance, dans le devenir, toute copie des archétypes intelligibles, une sorte de support nu, invisible et sans forme, apte seulement à recevoir l'empreinte des Idées, ainsi qu'à fournir espace et matière à la croissance de ce qui devient; il ne participe à l'intelligible que d'une façon très aporétique et très difficile à entendre, il n'est pas perceptible par les sens, mais par une sorte de raisonnement bâtard à peine croyable. Et pourtant ce "troisième genre" est indestructible et sans son concours le monde sensible ne s̀aurait exister par la seule puissance des Idées: il faut qu'existe quelque chose qui reçoive leur empreinte. Mais ce quelque chose est réduit à la plus extrême pauvreté. L'indétermination pure antérieure au Cosmos n'est même plus un chaos. C'est une sorte de matière première exténuée, toutefois étendue, en attente des Formes.

Anaxagore est un présocratique et un héritier des Ioniens, mais il est post-parménidien. Le Chaos du fond du Ciel, d'où procède toute chose, assure l'unité et l'éternité de l'être de la Nature en même temps que la possibilité du devenir du Cosmos et dans le Cosmos. Mais il faut que l'Intelligence cosmique, qui lui coexiste dans son unité, son infinité et son éternité, lui communique le mouvement qui révèlera sa richesse. 